\title{
Effects of a finite Dirac cone on the dispersion properties of graphite
}

\author{
Tim Gould and John F. Dobson \\ Qld Micro- and Nanotechnology Centre, Griffith University, Nathan, Qld 4111, Australia \\ S. Lebègue \\ Laboratoire de Cristallographie, Résonance Magnétique et Modélisations (CRM2, \\ UMR CNRS 7036) Institut Jean Barriol, Université de Lorraine BP 239, \\ Boulevard des Aiguillettes 54506 Vandoeuvre-lès-Nancy, France
}

\begin{abstract}
In a Letter by Lebègue et. al.[PRL 105, 196401 (2010)] describing benchmark ab initio "ACFDRPA" calculations of graphite, it was demonstrated that the dispersion energy of uniaxially stretched graphite obeyed a van der Waals power law of form $U^{\mathrm{vdW}}=-C_{3} D^{-3}$ where $D$ is the inter-layer distance, in agreement with earlier theoretical models. However, the coefficient was found to be $0.125 \mathrm{eV} \AA^{3} /$ atom in the calculations compared to $0.80 \mathrm{eV}^{3} /$ atom predicted by the theoretical models. In this work we show that much of this discrepancy can be explained by the false assumption in the theoretical model that the Dirac cones extended infinitely rather than being confined to a finite energy range, as in the numerical RPA work. We develop an improved model that takes into account this finite range via an imposed cutoff energy on transitions, and show that the dispersion energy is better represented by $U^{\mathrm{vdW}} \approx-C_{3} D^{-3}\left[\frac{2}{\pi} \operatorname{atan}\left(D / D_{c}+\phi\right)\right]$ where $C_{3}=0.38 \mathrm{eV} \AA^{3} /$ atom and $D_{c}$ and $\phi$ depend on the finite energy cutoff. This is of the same form as previously predicted for $D \rightarrow \infty$, but gives much better agreement with the ACFD-RPA results for appropriate values of the energy cutoff at intermediate layer spacings. The modified dispersion law will be important in the development of robust, general models of the inter-layer graphitic potential.
\end{abstract}

PACS numbers: 73.20.Mf,73.22.Pr,71.10.-w

\section{INTRODUCTION}

Following the initial isolation of monolayer graphene, ${ }^{1}$ and especially following Geim and Novoselov's seminal work ${ }^{2}$ graphene layers and bulk graphite have become one of the most studied problems in condensed matter physics. One of the many unusual properties of graphene is the presence of "Dirac cones" in its bandstructure. ${ }^{3,4}$ That is two bands (in fact two sets of two bands in the Brillioun zone) of conical shape whose tips meet at the Fermi energy. These cones are determined entirely by the $\pi_{z}$ orbitals via a combination of symmetry and band filling.

As a result of the Dirac cones and two-dimensional geometry, ${ }^{5}$ an unusual inter-layer van der Waals (vdW) dispersion potential between two graphene layers was predicted by Dobson et. al.. ${ }^{6}$ They showed that instead of the usual asymptotic attractive vdW potential $U^{\mathrm{vdW}}=-C_{4} D^{-4}$ between two insulating layers at a distance $D$, graphene was instead dominated by a $U^{\mathrm{vdW}}=-C_{3} D^{-3}$ power law, coming exclusively from the $\pi_{z}$ electrons. This was later ${ }^{7}$ extended to bulk graphite under uniaxial strain (where all layers are pulled apart equally to a distance $D$ ) where an identical power law was found, differing only in its $C_{3}$ coefficient. In the latter work it was predicted that the coefficient should be $C_{3}=0.80 \mathrm{eV}^{3} /$ atom, although this work used an incorrect expression for the response (discussed later) and should have predicted $C_{3}=0.39 \mathrm{eV \AA}^{3} /$ atom (with a Fermi velocity $v_{0}=570 \mathrm{~km} / \mathrm{s}$ ).

This power law was tested by Lebègue et. al. ${ }^{8}$ who investigated the properties of stretched bulk graphite via $a b$ initio adiabatic connection, fluctuation dissipation under the random-phase approximation (ACFD-RPA $)^{9-11}$ calculations using the VASP code. ${ }^{12}$ Their numerical experiment might be considered the present benchmark for the binding energy properties of graphite. While numerical issues made direct calculation of the asymptotic power law impossible, a simplified numerical RPA calculation was performed which retained only electronic transitions with an energy difference less than $1.25 \mathrm{eV}$, thereby restricting the response to low-energy $\pi_{z}$ transitions. This allowed use of a finer $\boldsymbol{k}$-space grid and isolated the $\pi_{z}$ part of the response from that due to other transitions, the latter being in fact dominant at the layer separations $D$ studied in the paper. ${ }^{8}$ This restricted numerical RPA calculation confirmed the analytically-predicted $D^{-3}$ power law. However the coefficient was found to be significantly smaller than that previously predicted at $C_{3}=0.125 \mathrm{eV \AA}^{3} /$ atom.

In this work we will show that the assumption by Dobson and co-workers that the Dirac cone in the bandstructure extended infinitely was responsible for much of this disagreement, being only valid in the extreme asymptotic limit $D \rightarrow \infty$ and being inappropriate at the separations up to $30 \AA$ where RPA numerics are feasible. By explicitly restricting transitions in the analytic approach, to match the restricted numerical RPA work, we will produce an improved model of the graphite response function for the $\pi_{z}$ orbitals (the response of the $\pi_{z}$ orbitals to changes in the external potential) and through it an improved vdW potential for graphite. This improved model is expected to better represent reality than the simple power law previously predicted. 


\section{GRAPHENE $\pi_{Z}$ RESPONSE}

It was shown by Dobson, White and Rubio ${ }^{6}$ that at large distance $D$, the dispersive energy of graphene is dominated by the density-density response of the $\pi_{z}$ orbitals only. By considering only those states that contribute to the Dirac cone, the averaged density-density response function $\chi_{0}(\boldsymbol{q} ; \omega)$ of the $\pi_{z}$ orbitals (at a given wavenumber $\boldsymbol{q}$ and frequency $\omega$ ) can be evaluated (see e.g. Refs. 13 and 14 for further details). Due to the layered nature of graphene, the response is essentially independent of the component of the wavenumber perpendicular to the plane, and we may thus consider only two dimensional wavenumbers in the plane so that $\boldsymbol{q} \equiv\left(q_{x}, q_{y}\right)$. We can thus approximate the response in the form of an integral over transitions between two Dirac-like cones (in two dimensions) centered at (and defining) the special $\mathrm{K}$ points, and with associated Fermi velocity gradients $v_{0}$.

\section{A. Infinite cones}

For small wavenumber $q=|\boldsymbol{q}|$ the total response of the $\pi_{z}$ orbitals at imaginary frequency $u$ is given by (see Appendix A for further details)

$$
-\chi_{0}(q ; i u) \approx 4 \int_{2 \mathrm{D}} \frac{\mathrm{d} \boldsymbol{k}}{(2 \pi)^{2}} \frac{2 \epsilon(k, p)}{\epsilon(k, p)^{2}+u^{2}}|L(\boldsymbol{k}, \boldsymbol{p})|^{2}
$$

in atomic units with $\hbar=e^{2} /\left(4 \epsilon_{0}\right)=m_{e-}=1$ used throughout this work. The factor four out the front of the integral is the result of summing over two cones with two spins each, and we integrate (choosing our origin to lie at a special $\mathrm{K}$ point) over transitions from orbitals with Bloch wavenumber $\boldsymbol{k}$ in the highest-occupied $\pi_{z}$ band, to orbitals with Bloch wavenumber $\boldsymbol{p}=\boldsymbol{k}+\boldsymbol{q}$ in the lowest-unoccupied band. Here $\epsilon(k, p) \equiv v_{0}(k+p)$ is the transition energy between the $\boldsymbol{k}$ and $\boldsymbol{p}$ orbitals, and $|L(\boldsymbol{k}, \boldsymbol{p})|^{2} \equiv(1-\hat{\boldsymbol{k}} \cdot \hat{\boldsymbol{p}}) / 2$ is the effective overlap of the occupied/unoccupied Bloch orbitals. The formula (1) appears in the above integral form in equation (30) of Dobson $^{14}$ (which must be doubled to account for the two K points) and equation (33) of Sodemann and Fogler ${ }^{15}$ (ignoring their screening term $\Sigma$ ).

By rotating our axes so that $\boldsymbol{q}=q \boldsymbol{e}_{x}$ lies on the $x$-axis, we can greatly simplify equation (1) by an elliptical coordinate transform to $\boldsymbol{k}=\frac{q}{2}(\cosh \mu \cos \nu-1, \sinh \mu \sin \nu)$ where $\mu \in[0, \infty), \nu \in[-\pi, \pi)$ and $\mathrm{d} \boldsymbol{k}=(q / 2)^{2}\left(\cosh ^{2} \mu-\right.$ $\left.\cos ^{2} \nu\right) \mathrm{d} \mu \mathrm{d} \nu$. It can be shown (see Appendix B) that $\epsilon=v_{0} q \cosh \mu$, and $L=\frac{\sin ^{2} \nu}{\cosh ^{2} \mu-\cos ^{2} \nu}$ and we can integrate over $\nu$ to rewrite (1) as

$$
\begin{aligned}
{ }_{\chi_{0}(q ; i u)} & =\frac{q^{2}}{2 \pi} \int_{0}^{\infty} \mathrm{d} \mu \frac{v_{0} q \cosh \mu}{u^{2}+\left(v_{0} q \cosh \mu\right)^{2}} \\
& \equiv \frac{q^{2}}{4 \sqrt{u^{2}+\left(v_{0} q\right)^{2}}}
\end{aligned}
$$

which is the familar ${ }^{15,16}$ expression for the $\pi_{z}$ response of graphite.

We note here that in earlier works Dobson et. al. ${ }^{6}$ and Gould et. al. ${ }^{7,17}$ used $\chi_{0}=-q^{2} /\left(2 \sqrt{u^{2}+\left(v_{0} q\right)^{2}}\right)$ rather than (3). This was caused by an arithmetic error in the derivation of the response and is responsible for the change from the previously published $C_{3}=$ $0.80 \mathrm{eV \AA} \AA^{3} /$ atom [via equation (8) of Ref. 7 with $v_{0}=$ $5.70 \mathrm{~km} / \mathrm{s}$ ] to $0.39 \mathrm{eVA}^{3} /$ atom.

\section{B. Restricting the transition energies}

In reality the Dirac cone is not infinite, as it a) is restricted to the Brillioun zone, b) is flattened out away from the special $\mathrm{K}$ points, and c) has higher order trigonal distortion. We can crudely model this by restricting our tranistion energies $\epsilon$ to be less than some cutoff energy $\epsilon_{c}$, and ignoring all other contributions. This restriction was made explicitly in the numerical calculations Lebègue et. al. ${ }^{8}$ to obtain the asymptotic results from which the $C_{3}$ coefficient was calculated. In that work calculation were performed with $\epsilon_{c}=1.25 \mathrm{eV}$. Clearly this approximation is an imperfect representation of reality, but it has the dual advantages of making derivation of the response function analytic, and allowing direct comparison with Ref. 8.

In our calculation of the response we must thus integrate only over those $\mu$ for which $\epsilon \equiv v_{0} q \cosh \mu \leq \epsilon_{c}$. We can now replace (2) by

$$
-\chi_{0}^{\epsilon_{c}}(q ; i u)=\frac{1}{2 \pi} q^{2} \int_{0}^{s_{c}} \frac{\mathrm{d} s}{s^{2}+\left[u^{2}+\left(v_{0} q\right)^{2}\right]}
$$

where $s=v_{0} q \sinh \mu$ and $s_{c}=\sqrt{\max \left[\epsilon_{c}^{2}-\left(v_{0} q\right)^{2}, 0\right]}$ coming from $\sinh ^{2} x=\cosh ^{2} x-1$, and the requirement that the integral must be zero for $v_{0} q \geq \epsilon_{c}$. Integrating (4) finally gives

$$
\chi_{0}^{\epsilon_{c}}=\chi_{0}\left(\frac{2}{\pi} \operatorname{atan} \frac{\sqrt{\max \left[\epsilon_{c}^{2}-\left(v_{0} q\right)^{2}, 0\right]}}{U}\right)
$$

where $\chi_{0}=-q^{2} /(4 U)$ and $U=\sqrt{u^{2}+\left(v_{0} q\right)^{2}}$ (this is very similar to the result derived by Dobson ${ }^{14}$ via a similar restriction). Here the cutoff is always sampled for sufficiently large $q$ and $u$, with important consequences for dispersion. Equation (5) should be considered a better representation of reality than (3) and is one of the more important results of this work.

\section{GRAPHITE DISPERSION IN THE RPA}

Gould et. $a l^{7}$ showed that, under the random-phase approximation, the dispersion energy of $\pi_{z}$ electrons in 
bulk graphite under uniaxial stretching obeys

$$
\begin{aligned}
U^{\mathrm{vdW}}= & \frac{v_{0}}{4 \pi^{2} D^{3}} \int_{0}^{1} \mathrm{~d} \lambda \int_{0}^{\infty} \theta^{2} \mathrm{~d} \theta \int_{0}^{\infty} \cosh \eta \mathrm{d} \eta \\
& \times\left[\mathcal{F}\left(\mathcal{C}^{\epsilon_{c}}\right)-\mathcal{F}_{\infty}\left(\mathcal{C}^{\epsilon_{c}}\right)\right]
\end{aligned}
$$

where

$$
\begin{aligned}
\mathcal{F}(\mathcal{C}) & =\frac{\mathcal{C}(\theta, \nu) \sinh \theta}{\sqrt{[\cosh \theta+\lambda \mathcal{C}(\theta, \nu) \sinh \theta]^{2}-1}}, \\
\mathcal{F}_{\infty}(\mathcal{C}) & \equiv \lim _{\theta \rightarrow \infty} \mathcal{F}(\mathcal{C})=\frac{\mathcal{C}(\theta, \nu)}{1+\lambda \mathcal{C}(\theta, \nu)}
\end{aligned}
$$

with the coordinate transformation $\theta=q D$ and $\sinh \eta=$ $u /\left(v_{0} q\right)$. Here we use the unitless $2 \mathrm{D}$ response

$$
\begin{aligned}
\mathcal{C}(\theta, \nu): & =\mathcal{C}\left(q=\theta / D, u=v_{0} q \sinh \nu\right) \\
& =-w_{2 D} \chi_{0}=-2 \pi q^{-1} \chi_{0}
\end{aligned}
$$

(where $w_{2 D}$ is the $2 \mathrm{D}$ Coulomb potential in reciprocal space) which governs the fluctuating interactions in the plane.

FIG. 1. Unitless response functions $\mathcal{C}(\theta, \eta)$ vs $\theta$ (with $\sinh \eta=$ 1) and $n$ (writh $\theta=0$ 1) Here $\left.\epsilon_{-}=1\right) 50 \mathrm{~V}$ m $=8.50 \mathrm{~km} / \mathrm{s}$ and
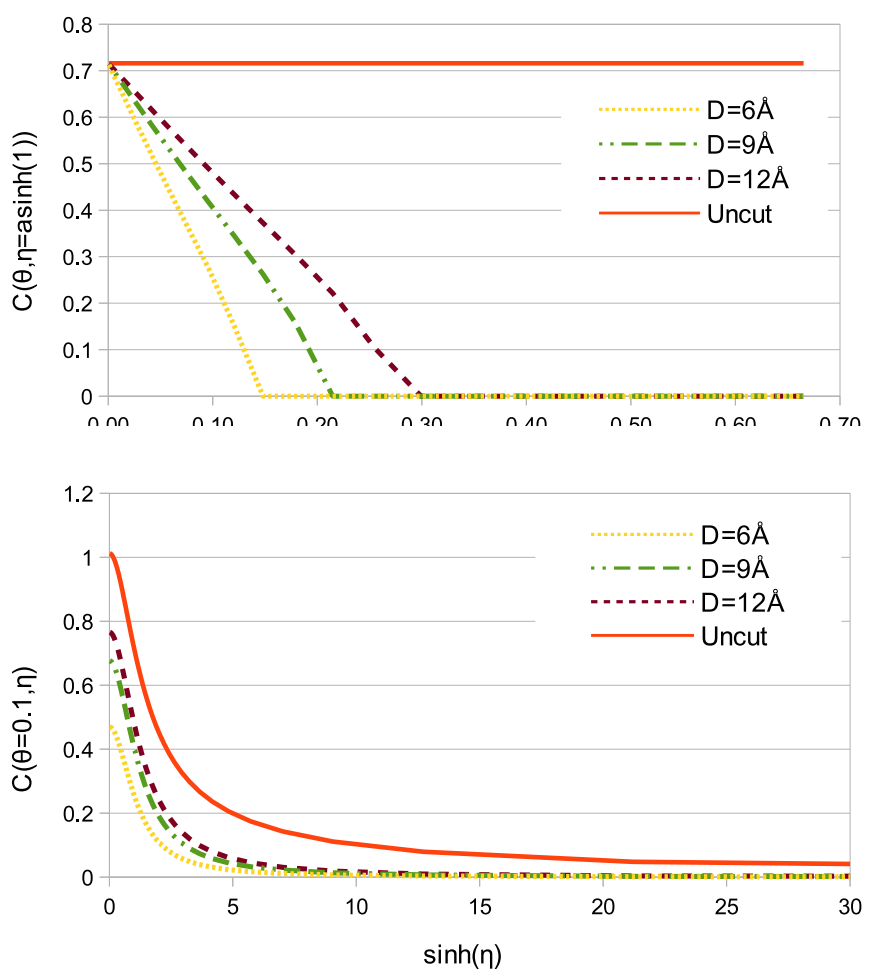

For the $\pi_{z}$ response of graphene without a cutoff it is easily shown ${ }^{7}$ that the unitless response $\mathcal{C}=\kappa / \cosh \eta$ $\left[\kappa=\pi /\left(2 v_{0}\right)\right.$ for $v_{0}$ in atomic units] is independent of $D$ and $\theta$ so that $D$ appears only outside the integral in (6) and the potential takes the simple form $U^{\mathrm{vdW}}=-C_{3}^{\infty} D^{-3}$. With a cutoff, this is no longer the case as now

$$
\mathcal{C}^{\epsilon_{c}}=\frac{\kappa}{\cosh \eta}\left[\frac{2}{\pi} \operatorname{atan} \frac{\sqrt{\max \left[\theta_{c}^{2} / \theta^{2}-1,0\right]}}{\cosh \eta}\right]
$$

explicitly depends on $D$ via $\theta_{c}=\epsilon_{c} D / v_{0}$. In Figure 1 we plot the unitless response given by equation (10) for graphite with interlayer distances $D=6,9,12 \AA$. We use $\epsilon_{c}=1.25 \mathrm{eV}$ and $v_{0}=850 \mathrm{~km} / \mathrm{s}$ so that $\kappa=4.05$ and $\theta_{c}=0.134,0.201,0.268$. The chief effect of the $\theta=q D$ dependence is to introduce a near linear fit down to a $D$ dependent cutoff. The second plot illustrates one of the advantages of the cutoff. For large $u=v_{0} q \sinh \eta$ the response decays correctly with $u^{-2}$, whereas without a cutoff it decays only with $u^{-1}$

The correction introduces a further dependence on $D$ to the dispersion law, and we must write the dispersion law as

$$
\begin{aligned}
U^{\mathrm{vdW}}= & \frac{-C_{3}^{\epsilon_{c}}(D)}{D^{3}} \\
C_{3}^{\epsilon_{c}}(D)= & \frac{v_{0}}{4 \pi^{2}} \int_{0}^{1} \mathrm{~d} \lambda \int_{0}^{\infty} \theta^{2} \mathrm{~d} \theta \int_{0}^{\infty} \cosh \eta \mathrm{d} \eta \\
& \times\left[\mathcal{F}_{\infty}\left(\mathcal{C}^{\epsilon_{c}}\right)-\mathcal{F}\left(\mathcal{C}^{\epsilon_{c}}\right)\right] .
\end{aligned}
$$

Since we integrate functions of $\mathcal{C}^{\epsilon_{c}}$ over all $\theta$ and $\eta$ to find $C_{3}^{\epsilon_{c}}(D)$, it is apparent that the cutoff will be quite important to the dispersion energy. Aside from the integral over $\lambda$, equation (12) must be evaluated via quadrature, and is unlikely to have a closed form representation outside the very far asymptotic regime where it reduces to the previously found constant $C_{3}^{\infty}$.

However some insight into the approximate form of the power law can be gained from the $\theta$ integral in (12). To leading order, its integrand has an effective weight $\theta^{2} e^{-2 \theta}$ and is thus dominated by $\theta \approx 1$ ( or $q \approx 1 / D$ ). Assuming a slow variation of the arctangent in $C^{\epsilon_{c}}$ this suggests that the effect of the restricted transitions on the dispersion potential (11) should be well approximated by

$$
U^{\mathrm{vdW}} \approx \frac{-C_{3}^{\infty}}{D^{3}}\left[\frac{2}{\pi} \operatorname{atan}\left(\frac{D}{D_{c}}+\phi\right)\right] .
$$

Here $C_{3}^{\infty}$ is the $C_{3}$ coefficient using the 'uncut' $\chi$ and valid in the $D \rightarrow \infty$ limit, while fitting parameters $D_{c}$ and $\phi$ are found by minimising the error of (13) compared to (6). Parameter $D_{c}$ depends approximately on the cutoff energy $\epsilon_{c}$ and velocity $v_{0}$ via $D_{c} \propto v_{0} / \epsilon_{c}$, while $\phi$ takes into account the more complicated effects of the $D$ dependent integrand. Numerical tests confirm the validity of equation (13), and it is the principal result of this work, showing the explicit correction to the van der Waals coefficient from the finite cutoff.

\section{NUMERICAL RESULTS}

In our previous work ${ }^{7}$ we used $v_{0}=570 \mathrm{~km} / \mathrm{s}$ to find $C_{3}^{\infty}=0.80 \mathrm{meV}^{3} /$ atom with the incorrect $\chi_{0}=$ 
$q^{2} /(2 U)$ used in that work. Using the correct response $\chi_{0}=q^{2} /(4 U)$ and $v_{0}=570 \mathrm{~km} / \mathrm{s}$ instead gives $C_{3}^{\infty}=$ $0.39 \mathrm{meV}^{3}$ /atom. In this work we shall instead use the Fermi velocity $v_{0}=850 \mathrm{~km} / \mathrm{s}$ found from the VASP LDA band structure, which gives $C_{3}^{\infty}=0.38 \mathrm{meV}^{3} /$ atom. The small change from 0.39 to 0.38 of $C_{3}^{\infty}$ (with $\chi_{0}=$ $\left.q^{2} /(4 U)\right)$ comes entirely from the different choice of $v_{0}$.

FIG. 2. $\pi_{z}$ dispersion energy for a variety of finite cutoffs, and the 'uncut' theory with $v_{0}=850 \mathrm{~km} / \mathrm{s}$. RPA results taken from Ref. 8.

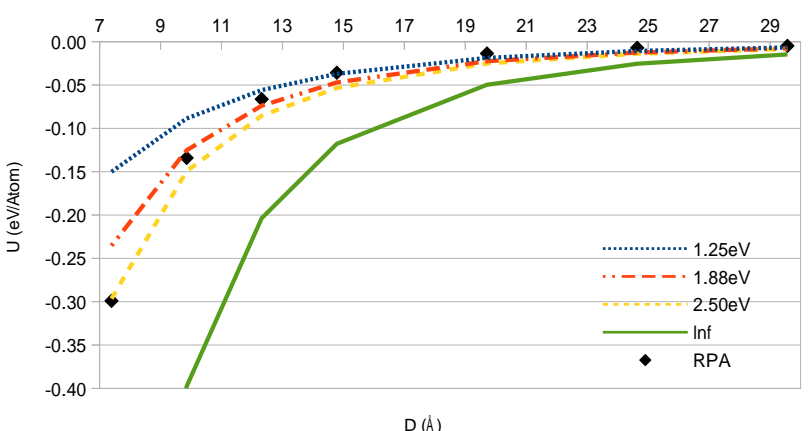

In Figure 2 we plot potential energy curves using the ACFD-RPA data points from Ref. 8 along with results from the traditional uncut model giving $U^{\mathrm{vdW}}=$ $-C_{3}^{\infty} D^{-3}$, and the new model with a cutoff calculated via (6). It is immediately clear that the energy results using the uncut model are quite poor when compared with the ACFD-RPA results, while those with the new model with a finite cutoff are significantly better. For our finite cutoffs we choose $\epsilon_{c}=1.25 \mathrm{eV}$ to match the original ACFD-RPA calculations for $C_{3}$ which were evaluated with this transition cutoff. We also include some larger cutoffs to account for numerical and other effects. As desired, good agreement in the tail $D \gtrsim 12 \AA$ is found with $\epsilon_{c}=1.25 \mathrm{eV}$, while the inner regions $D \lesssim 12 \AA$ are better matched by higher effective cutoffs.

There are a number of potential causes for the disagreement between the model (with $\epsilon_{c}=1.25 \mathrm{eV}$ ) and the $a b$ initio calculations, especially: i) a failure of the theory to model imperfections in the shape of the cone in the real band-structure, and related effects on the overlap $|L|^{2}$, ii) local-field effects ${ }^{14}$ being ignored in the model interacting response, and iii) numerical issues (discussed below). We expect effect i) to be dominant, and to come mostly from the trigonal warping from higher-order effects. ${ }^{18}$ These imperfections will have an effect on the response function, and through it the dispersion energy. Furthermore, the magnitude of this effect will be greatest for smaller $D$, where larger wavenumbers are sampled so that deviations from the ideal conical shape are weighted more heavily in the integrals. Similarly the local-field effects ii) will contribute more in the smaller $D$ limit, as here the "local field error" on reciprocal lattice vector $\boldsymbol{G}$ approximately decays as $e^{-|\boldsymbol{q}+\boldsymbol{G}| D} / e^{-q D}$, and is thus magnified for smaller $D$ (see Ref. 14).
TABLE I. Cutoff dependent fitting parameters $D_{c}$ and $\phi$ for the dispersion formula $U^{\mathrm{vdW}}=-C_{3}^{\infty} D^{-3} \frac{2}{\pi} \operatorname{atan}\left(D / D_{c}+\phi\right)$. Here we use $v_{0}=850 \mathrm{~km} / \mathrm{s}$ so that $C_{3}^{\infty}=0.38 \mathrm{eV} \AA^{3} /$ atom.

\begin{tabular}{l|rrrrrrrr}
\hline \hline$\epsilon_{c}(\mathrm{eV})$ & 0.5 & 1.0 & 1.25 & 1.5 & 2.0 & 2.5 & 3.0 & 5.0 \\
$D_{c}(\AA)$ & 55.4 & 39.5 & 37.7 & 36.5 & 34.3 & 32.0 & 30.0 & 23.7 \\
$\phi$ & -0.08 & 0.05 & 0.12 & 0.19 & 0.30 & 0.38 & 0.44 & 0.62 \\
\hline \hline
\end{tabular}

Regarding the numerical work, the results presented in Ref. 8 remain slightly dependent on several parameters affecting the present context. These include: finite 'smearing' at the Fermi energy, finite number of $\boldsymbol{k}$-points for the various integrations over the Brillouin zone, finite number of frequencies used for the calculation of the RPA correlation energy, and an extrapolation to infinite size for the dielectric function. A convergence of about $1 \mathrm{meV}$ /atom was obtained for the binding energy in Ref. 8, but this was reduced for the energy differences used to evaluate the $\pi_{z}$ dispersion by improved convergence parameters. As such, it is likely that points i) and ii) will present the dominant source of difference.

Returning to general theory, we fit equation (13) to numerically evaluated dispersion energies calculated via equation (6), to determine fitting parameters $D_{c}$ and $\phi$ for a variety of cutoff energies. We restrict ourself to the LDA Fermi velocity $v_{0}=850 \mathrm{~km} / \mathrm{s}$, appropriate to the numerical VASP calculation, where $C_{3}^{\infty}=$ $0.38 \mathrm{eV \AA} \AA^{3} /$ atom, but interpolation to other velocities should be straightforward as $D_{c} \propto v_{0} / \epsilon_{c}$. The correction due to the energy cutoff is quite significant, and even for a cutoff of $5.0 \mathrm{eV}$ close to the full width of the Dirac cones, offers a reduction of $30 \%$ on the usual $C_{3}$ coefficient at $30 \AA$, and $55 \%$ at $3.34 \AA$. For comparison the correction found by Sernelius ${ }^{19}$ to account for finitewidth slabs takes an absolute maximum of $-30 \%$ near contact.

\section{CONCLUSIONS}

We have shown in this work that the Dirac cones should not be considered to be of infinite span for the prediction of van der Waals dispersion of stretched graphite, especially in the 'middle' range of distances where the magnitude of the dispersion interaction is significant. The importance of the finite cones comes from the fact that the dispersion energy samples the density response at all wavenumbers $q$ and imaginary frequencies $u$, albeit with greatest weighting on $q \approx 1 / D$ where $D$ is the inter-layer distance. We note that any other properties of graphite that depend on a finite frequency and wavenumber should not trivially assume an infinite cone either.

By including a cut-off we are better able to reproduce the 'reality' of the ACFD-RPA calculations, through the modified vdW energy equation (13). The large difference between $a b$ initio theory, and the dispersion model found 
by Lebègue et. al. ${ }^{8}$ can now be mostly resolved by the correction to $\chi_{0}$. Precise agreement between the model and calculations could not be found, especially in the near contact region. However differences can be easily explained by neglect of the non-ideal geometry of the cones in real graphite, a failure to account for local field effects of intra-planar electric screening in the model, and numerical limitations in the ACFD-RPA.

While we have focused mainly on an artifical cutoff on the transitions, imposed to make analysis easy and numerical calculations tractable, the effect is certainly representative of reality, albeit it with a larger, smeared cutoff. We thus consider it likely that equation (13) is a good representation of reality for some $D_{c}$ and $\phi$, and that it will remain the dominant dispersion component for sufficiently large distance $D$. We thus recommend that any explicit addition of $\pi_{z}$ dispersion corrections such as that used by Totton, Misquitta and $\mathrm{Kraft}^{20}$ use $U^{\mathrm{vdW}}=-C_{3} D^{-3}\left[\frac{2}{\pi} \operatorname{atan}\left(D / D_{c}+\phi\right)\right]$ with $D_{c}$ and $\phi$ chosen appropriately, rather than $U^{\mathrm{vdW}}=-C_{3} D^{-3}$.

The effect of the finite cutoff on van der Waals laws is not restricted to changing the dispersive binding properties of bulk graphite. It will also affect the magnitude of the dispersive power laws of cleavage and exfoliation in graphite, ${ }^{17}$ while leaving the exponents unchanged. In those cases, however, we expect the affect to be weak as inter-layer screening is likely to reduce the contribution of the finite cone to energies, and the $\pi_{z}$ electrons already contribute only a very small fraction of the total dispersion. We thus consider it likely that the effect of a finite cone can be ignored when considering cleavage and exfoliation.

\section{ACKNOWLEDGMENTS}

TG and JFD were supported by Australian Research Council Discovery Grant DP1096240. SL acknowledges financial support from the Université de Lorraine through the program "Soutien à la dimension internationale de la recherche".

\section{Appendix A: Graphene response}

It can be readliy shown that the response function (integrated over a unit cell $\mathcal{U}$ ) of any many-electron bulk system expanded on Bloch orbitals

$$
\phi_{m \boldsymbol{k}}(\boldsymbol{r})=\sum_{\boldsymbol{G}} c_{m \boldsymbol{k}}(\boldsymbol{G}) e^{i(\boldsymbol{G}+\boldsymbol{k}) \cdot \boldsymbol{r}} \equiv e^{i \boldsymbol{k} \cdot \boldsymbol{r}} u_{m \boldsymbol{k}}(\boldsymbol{r})
$$

is given as (for $N_{s}=2$ spins)

$$
\begin{aligned}
-\chi_{0}(\boldsymbol{q})= & N_{s} \int_{\mathrm{BZ}} \frac{\mathrm{d} \boldsymbol{k}}{(2 \pi)^{3}} \sum_{m n} f_{m \boldsymbol{k}}\left(1-f_{n \boldsymbol{p}}\right) \\
& \times \frac{2\left(\epsilon_{m \boldsymbol{k}}-\epsilon_{n \boldsymbol{p}}\right)}{\left(\epsilon_{m \boldsymbol{k}}-\epsilon_{n \boldsymbol{p}}\right)^{2}+u^{2}}\left|L_{m \boldsymbol{k}, n \boldsymbol{p}}\right|^{2} \\
\left|L_{m \boldsymbol{k}, n \boldsymbol{p}}\right|^{2}= & \left|\int_{\mathcal{U}} \mathrm{d} \boldsymbol{r} e^{i \boldsymbol{q} \cdot \boldsymbol{r}} u_{m \boldsymbol{k}}^{*}(\boldsymbol{r}) u_{m \boldsymbol{p}}(\boldsymbol{r})\right|^{2} \\
= & \left|e^{i \boldsymbol{q} \cdot \boldsymbol{r}} \sum_{\boldsymbol{G}} c_{m \boldsymbol{k}}^{*}(\boldsymbol{G}) c_{n \boldsymbol{p}}(\boldsymbol{G})\right|^{2}
\end{aligned}
$$

where $m$ and $n$ label the bands, $\boldsymbol{p}=\boldsymbol{k}+\boldsymbol{q}$, and $f_{m \boldsymbol{k}}$ is one if the Bloch energy $\epsilon_{m \boldsymbol{k}}$ is less than the Fermi energy $\epsilon_{f}$ or zero otherwise. Transitions are integrated over the Bloch wavenumbers $\boldsymbol{k}$ in the Brillioun sone (BZ). For the purpose of this work we deal with effectively $2 \mathrm{D}$ graphene sheets restricted to the $\pi_{z}$ orbitals only. For this case

$$
\begin{aligned}
-\chi_{0}(\boldsymbol{q}) & =2 N_{K} \int_{\mathrm{BZ}^{\prime}} \frac{\mathrm{d} \boldsymbol{k}}{(2 \pi)^{3}} \frac{2 \epsilon_{\boldsymbol{k}, \boldsymbol{p}}}{\epsilon_{\boldsymbol{k}, \boldsymbol{p}}^{2}+u^{2}}|L(\boldsymbol{k}, \boldsymbol{p})|^{2} \\
|L(\boldsymbol{k}, \boldsymbol{p})|^{2} & =2 \pi \delta\left(q_{z}\right)\left|e^{i \boldsymbol{q} \cdot \boldsymbol{r}} \sum_{\boldsymbol{G}} c_{\mathrm{H} \boldsymbol{k}}(\boldsymbol{G}) c_{\mathrm{L} \boldsymbol{p}}^{*}(\boldsymbol{G})\right|^{2}
\end{aligned}
$$

where we have restricted the sum to transitions (with energy $\epsilon_{\boldsymbol{k}, \boldsymbol{p}}$ ) from the highest occupied (H) to lowest unoccupied (L) orbital bands in a sub-region BZ' of the Brillioun zone containing only a single special $\mathrm{K}$ point in the integral, and used the $N_{K}$ symmetries to evaluate the other $\mathrm{K}$ point(s).

Making a tight-binding model of the $\pi_{z}$ orbitals allows us to derive analytic expressions (see e.g. sections 2.1-2.4 of Ref. 14 for a derivation)

$$
\begin{aligned}
\epsilon_{\boldsymbol{k}, \boldsymbol{p}} \equiv \epsilon(k, p) & =v_{0}(k+p) \\
|L(\boldsymbol{k}, \boldsymbol{p})|^{2} & =\frac{1}{2}(1-\hat{\boldsymbol{k}} \cdot \hat{\boldsymbol{p}})
\end{aligned}
$$

when $q$ is small and $\boldsymbol{k}$ is close to a special K point. Thus, for a $\pi_{z}$ restricted graphitic system with $N_{K}$ Dirac cones in the $\mathrm{BZ}$ we can write the response function as:

$$
-\chi_{0}=2 N_{K} \int_{2 D} \frac{\mathrm{d} \boldsymbol{k}}{(2 \pi)^{2}} \frac{2 \epsilon(k, p)}{\epsilon(k, p)^{2}+u^{2}}|L(\boldsymbol{k}, \boldsymbol{p})|^{2}
$$

where we have replaced the restricted integral by one extending to infinite. With $N_{K}=2$, this expression is identical to equation (3) of Ref. 21 using their $|L|^{2}=$ $4 \sin ^{2}(\Delta / 2)=4(1-\hat{\boldsymbol{k}} \cdot \hat{\boldsymbol{p}}) / 2$ where $\Delta$ is the angle between $\boldsymbol{k}$ and $\boldsymbol{p}$. However further inspection (below) suggests that equation (4) of the same work appears to have an extra factor of two in front, which has erroneously carried through to Refs. 6, 7, and 17.

With a change of variables to $k=q / 2(\cosh \mu \cos \nu-$ $1, \sinh \mu \sin \nu)$ with $\mu \in[0, \infty), \nu \in[-\pi, \pi)$ (see Appendix $\mathrm{B}$ for details) this becomes

$$
-\chi_{0}=\frac{2 N_{K}}{4 \pi^{2}} \int \frac{q^{2} X \mathrm{~d} \mu \mathrm{d} \nu}{4} \frac{\sin ^{2} \nu}{X} \frac{2 v_{0} q \cosh \mu}{\left(v_{0} q \cosh \mu\right)^{2}+u^{2}}
$$


where we cancel $X=\cosh ^{2} \mu-\cos ^{2} \nu$ top and bottom. Integrating over $\nu$ gives

$$
\begin{aligned}
-\chi_{0} & =\frac{2 N_{K} q^{2}}{8 \pi} \int_{0}^{\infty} \mathrm{d} \mu \frac{q v_{0} \cosh \mu}{\left(q v_{0} \cosh \mu\right)^{2}+u^{2}} \\
& =\frac{2 N_{K} q^{2}}{8 \pi} \frac{\pi}{2 \sqrt{v_{0}^{2} q^{2}+u^{2}}}=\frac{N_{K} q^{2}}{8 \sqrt{v_{0}^{2} q^{2}+u^{2}}}
\end{aligned}
$$

which finally gives $-\chi_{0}=q^{2} /\left(4 \sqrt{v_{0}^{2} q^{2}+u^{2}}\right)$ for an isolated Graphene layer with two infinite cones and two spins.

\section{Appendix B: Change of variables}

In their paper, Sodemann and Fogler ${ }^{15}$ proposed a judicious change of variables from Cartesian coordinates $k_{x}, k_{y}$ to ellipitcal coordinates $\mu, \nu$ for $\mu \in[0, \infty)$ and $\nu \in$ $[-\pi, \pi)$ that makes evaluation of the response integrals in this work significantly simpler. Under this transformation we set $\boldsymbol{q}=(q, 0), \boldsymbol{k}=\frac{q}{2}(\cosh \mu \cos \nu-1, \sinh \mu \sin \nu)$ and $\boldsymbol{p}=\frac{q}{2}(\cosh \mu \cos \nu+1, \sinh \mu \sin \nu)$. The integration is performed over

$$
\begin{aligned}
\mathrm{d} \boldsymbol{k} & \equiv \mathrm{d} k_{x} \mathrm{~d} k_{y}=\left(\frac{\partial k_{x}}{\partial \mu} \frac{\partial k_{y}}{\partial \nu}-\frac{\partial k_{x}}{\partial \nu} \frac{\partial k_{y}}{\partial \mu}\right) \mathrm{d} \mu \mathrm{d} \nu \\
& =\frac{q^{2}}{4}\left(\cosh ^{2} \mu \sin ^{2} \nu+\sinh ^{2} \mu \cos ^{2} \nu\right) \mathrm{d} \mu \mathrm{d} \nu \\
& =\frac{q^{2}}{4}\left(\cosh ^{2} \mu-\cos ^{2} \nu\right) \mathrm{d} \mu \mathrm{d} \nu
\end{aligned}
$$

where here and hence we use the identities

$$
\begin{aligned}
& \cosh ^{2} \mu \sin ^{2} \nu+\sinh ^{2} \mu \cos ^{2} \nu=\cosh ^{2} \mu-\cos ^{2} \nu \\
& \sinh ^{2} \mu \sin ^{2} \nu+\cosh ^{2} \mu \cos ^{2} \nu=\cosh ^{2} \mu+\cos ^{2} \nu-1 .
\end{aligned}
$$

to eliminate sin and sinh terms.

We can now derive the important quantities

$$
\begin{aligned}
|k|= & \frac{q}{2}\left(\sinh ^{2} \mu \sin ^{2} \nu+\cosh ^{2} \mu \cos ^{2} \nu+1\right. \\
& -2 \cosh \mu \cos \nu)^{1 / 2} \\
= & \frac{q}{2} \sqrt{\cosh ^{2} \mu+\cos ^{2}-2 \cosh \mu \cos \nu} \\
= & \frac{q}{2}(\cosh \mu-\cos \nu) \\
|p|= & \frac{q}{2}(\cosh \mu+\cos \nu)
\end{aligned}
$$

(noting that $\cosh \mu \geq \cos \nu$ ) and

$$
\begin{aligned}
\boldsymbol{k} \cdot \boldsymbol{p} & =\frac{q^{2}}{4}\left(\sinh ^{2} \mu \sin ^{2} \nu+\cosh ^{2} \mu \cos ^{2} \nu-1\right) \\
& =\frac{q^{2}}{4}\left(\cosh ^{2} \mu+\cos ^{2} \nu-2\right) .
\end{aligned}
$$

Putting all these relationships together we finally show

$$
\begin{gathered}
\epsilon=v_{0}(|k|+|p|)=q v_{0} \cosh \mu \\
|L|^{2}=\frac{k p-\boldsymbol{k} \cdot \boldsymbol{p}}{2 k p}=\frac{\sin ^{2} \nu}{\cosh ^{2} \mu-\cos ^{2} \nu}
\end{gathered}
$$

which can be used in integral expressions together with (B1).

\section{REFERENCES}

${ }^{1}$ K. S. Novoselov, A. K. Geim, S. V. Morozov, D. Jiang, Y. Zhang, S. V. Dubonos, I. V. Grigorieva, and A. A. Firsov, Science 306, 666 (2004).

2 A. K. Geim and K. S. Novoselov, Nat Mater 6, 183 (2007).

3 P. R. Wallace, Phys. Rev. 71, 622 (1947).

${ }^{4}$ M. Klintenberg, S. Lebègue, C. Ortiz, B. Sanyal, J. Fransson, and O. Eriksson, Journal of Physics: Condensed Matter 21, 335502 (2009).

5 J. F. Dobson and T. Gould, Journal of Physics: Condensed Matter 24, 073201 (2012).

6 J. F. Dobson, A. White, and A. Rubio, Phys. Rev. Lett. 96, 073201 (2006).

7 T. Gould, K. Simpkins, and J. F. Dobson, Phys. Rev. B 77, 165134 (2008).

8 S. Lebègue, J. Harl, T. Gould, J. G. Ángyán, G. Kresse, and J. F. Dobson, Phys. Rev. Lett. 105, 196401 (2010).

9 H. Eshuis, J. Bates, and F. Furche, Theoretical Chemistry Accounts: Theory, Computation, and Modeling (Theoretica Chimica Acta) 131, 1084 (2012).
10 X. Ren, P. Rinke, C. Joas, and M. Scheffler, Journal of Materials Science, 7447 (2012).

11 J. F. Dobson, in Fundamentals of Time-Dependent Density Functional Theory, Lecture Notes in Physics, Vol. 837, edited by M. A. Marques, N. T. Maitra, F. M. Nogueira, E. Gross, and A. Rubio (Springer Berlin Heidelberg, 2012) pp. $417-441$.

12 G. Kresse and J. Furthmüller, Phys. Rev. B 54, 11169 (1996); G. Kresse and D. Joubert, ibid. 59, 1758 (1999).

13 K. W. K. Shung, Phys. Rev. B 34, 979 (1986).

14 J. F. Dobson, Surface Science 605, 1621 (2011).

15 I. Sodemann and M. M. Fogler, Phys. Rev. B 86, 115408 (2012).

16 E. H. Hwang and S. Das Sarma, Phys. Rev. B 75, 205418 (2007).

17 T. Gould, E. Gray, and J. F. Dobson, Phys. Rev. B 79, 113402 (2009).

18 T. Ando, T. Nakanishi, and R. Saito, Journal of the Physical Society of Japan 67, 2857 (1998). 
19 B. E. Sernelius, Graphene 01, 21 (2012).

20 T. S. Totton, A. J. Misquitta, and M. Kraft, The Journal of Physical Chemistry A 115, 13684 (2011).
${ }^{21}$ J. F. Dobson and A. Rubio, arXiv:cond-mat/0502422 (2005). 